\title{
Concepción de aprendizaje en estudiantes universitarios de la Carrera de Psicología del extremo norte de Chile*
}

\section{Psychology Undergraduates Learning Conception's from the} Chilean North

\author{
José Sebastián Sandoval Díaz ${ }^{\mathrm{a}}$ \\ Universidad de Atacama, Chile \\ ORCID: http://orcid.org/0000-0001-7247-7113 \\ Daniel IVÁN Pérez Zapata \\ Universidad de Tarapacá, Chile
}

aAutor de correspondencia. Correo electrónico: jose.sandoval@uda.d

Para citar este artículo: Sandoval, J. S., \& PérezZapata, D. I. (2017). Concepción de aprendizaje en estudiantes universitarios de la Carrera de Psicología del extremo norte de Chile. Universitas Psychologica, 16(2), 1-11.

https://10.11144/Javeriana.upsy16-2.caeu

\section{RESUMEN}

El presente trabajo busca determinar la influencia de la utilización de estrategias metacognitivas en relación con el nivel de pericia en psicología sobre la concepción de aprendizaje de estudiantes universitarios. Participaron 144 estudiantes de ambos sexos, pertenecientes a la ciudad de Arica (Chile). Se utilizó el cuestionario de estrategias metacognitivas (O’Neil \& Abedi, 1996) y el inventario de concepciones de aprendizaje (Martínez, 2004). Los resultados apuntan a que el nivel de pericia y las estrategias metacognitivas correlacionan significativamente con concepciones de aprendizaje. Sin embargo, en un análisis detallado para cada tipo de concepción, se encontraron diferencias estadísticamente significativas. Estos resultados evidencian que las concepciones del tipo constructiva e interpretativo se ven mayormente influenciadas por el uso de estrategias metacognitivas de aprendizaje, en contraposición al tipo de concepción directivo, el cual es explicado, en mayor medida, por el nivel de pericia inicial en el que se encuentran los estudiantes universitarios.

Palabras clave

estudiantes universitarios; concepciones de aprendizaje; nivel de pericia en psicología y estrategias metacognitivas

\footnotetext{
ABSTRACT

The following article aims to determine the influence of using metacognitive strategies related to perish levels of psychology on learning conceptions of psychology undergraduates. 144 subjects from a north city in Chile participated in this study. It was administered the Questionnaire of Metacognitive Strategies (O'Neil \& Abedi, 1996) and the Learning Conceptions Inventory (Martinez, 2004). Results showed significant correlations between learning conceptions with perish level and metacognitive strategies. However, a detailed analysis found significant results for each type of conception. These outcomes suggest that constructive and interpretative conceptions are greater influenced by the use of metacognitive strategies of learning, opposite to directive conception, which is explained, in a greater way, by levels of initial perish of psychology undergraduates.

Keywords

learning conceptions; perish levels; metacognitive strategies; psychology undergraduates
} 


\section{Introducción}

En las últimas décadas, desde las ciencias de la educación, ha surgido una serie de trabajos ligados al estudio del aprendizaje vía cambio conceptual. Esta área profundiza en la construcción cognitiva de conceptos, en adelante concepciones, en disciplinas tan variadas como la biología, la física, las humanidades, las ciencias sociales, por mencionar solo algunas. Estas concepciones se organizan bajo la forma de teorías personales, las cuales presentan grados diferenciales de estructuración formal (Carey, 1985a, 1985b). A partir de esto, el presente enfoque se ocupa tanto de las concepciones intuitivas, es decir, aquellas que presentan un menor grado de estructuración, como aquellas que presentan un alto grado de estructuración formal, examinando el proceso y los mecanismos de transformación o relaboración que ambos tipos presentan (Dysktra, Boyle, \& Monarch, 1992; Hewson \& Thorley, 1989; Kuhn \& Lao, 1998; Limón, 2001; Rodríguez Moneo \& Carretero, 1996; White \& Gunstone, 1989).

Las investigaciones aplicadas al contexto educativo en concepciones intuitivas postulan que detrás de las estrategias de aprendizaje empleadas por el estudiantado existiría un cuerpo teórico configurado a partir de un conjunto de supuestos de ideas incoherentes o inconexas entre sí (Säljö, 1979). Estos supuestos inconexos orientarían el logro de objetivos de aprendizaje, evidenciando el rol de las creencias como elemento de disposición antes de la acción (Pintrich, Marx, \& Boyle, 1993).

Respecto del proceso vinculado al desarrollo conceptual, en un momento inicial, las concepciones intuitivas tienden a configurarse a un nivel elemental de copia fiel del objeto por representar, es decir, de aprendizaje de tipo fijo, modificándose con posterioridad y gradualidad en modelos de mayor estructuración formal. Entre las estrategias y actitudes estudiantiles que facilitarían el enriquecimiento o restructuración de este tipo de concepciones, se han identificado la acción constructiva, la relatividad del pensamiento, el cambio en las ideas y la disposición al cambio (Tynjälä, 1997, 1999).

\section{Tipos de concepciones de aprendizaje}

Respecto del aprendizaje, Pozo y Scheuer (1999) proponen tres tipos de concepciones: (a) el directo, (b) el interpretativo y (c) el constructivo, los cuales se definirán a continuación.

El tipo de aprendizaje directo (a) considera que existe una correspondencia directa entre las condiciones de la enseñanza y el resultado de lo aprendido, partiendo del supuesto de que el conocimiento sería un reflejo fiel de la realidad. El tipo de aprendizaje interpretativo (b), por otro lado, plantea que la implicancia personal del aprendiz, a lo largo del proceso de enseñanza, se torna una actividad y estrategia imprescindible para el logro de objetivos, mediatizando la relación entre condiciones de aprendizaje y resultados esperados. Por último, el aprendizaje constructivo (c) asume como esencial los procesos continuos, relacionales y conflictivos entre sujeto-objeto, atribuyendo a estos procesos un rol mediacional en la reelaboración y transformación de lo aprendido.

Respecto de estos tres tipos de concepciones de aprendizaje y su correspondiente vínculo con el nivel educativo o ciclo vital del estudiantado, la evidencia empírica no ha sido concluyente. Por un lado, las investigaciones señalan la presencia de concepciones de alta estructuración formal tanto en estudiantes universitarios como en adolescentes; sin embargo, algunos trabajos señalarían la presencia de este tipo también en niños de 8 años (Pozo \& Scheuer, 1999). Contrario a esto, el tipo de aprendizaje directo también ha sido observado en personas adultas (Máiquez, Rodrigo, Capote, \& Vermaes, 2000), universitarios e incluso en personas dedicadas a la enseñanza formal (Pozo \& De la Cruz, 2003).

\section{Nivel de pericia}

El nivel de pericia se aboca al estudio sobre el dominio específico, en parcelas particulares del conocimiento, por parte de expertos o aprendices. Para Reif y Larkin (1991), el nivel de pericia se define como "la colección de conocimiento declarativo y procedimental 
necesario para atender a metas particulares, lo que implica el uso de conceptos específicos, las relaciones entre ellos y los métodos empleados en el mismo" (p. 735).

Por su parte, Pozo (2003) hace referencia a la noción de dominio específico del conocimiento como "un conjunto de sucesos o contextos que procesamos de acuerdo con las mismas restricciones o principios" (p. 201).

A partir de esto, desde el enfoque del dominio específico, y particularmente desde el nivel de pericia en la psicología como disciplina, se ha postulado que a medida que aumenta la experticia sobre esta materia también se incrementaría el dominio sobre los procesos cognitivos asociados al aprendizaje, siendo posible identificar niveles más complejos en dichas concepciones y en las estrategias de aprendizaje empleadas (Glaser, 1982).

En cuanto a la relación entre los tipos de concepciones de aprendizaje y el nivel de pericia en el dominio específico universitario, Schommer (1993; Schommer, Calvert, Gariglietti, \& Bajaj, 1997) encontraron la presencia de diferencias significativas en los supuestos epistemológicos sobre el aprendizaje que sostenían estudiantes universitarios pertenecientes a distintos niveles de formación disciplinar. Estos resultados convergen con estudios que han encontrado evidencia similar (Alexander, Murphy, Guan, \& Murphy, 1998; Boulton-Lewis, Marton, Lewis, \& Wilss, 2000; Boulton-Lewis, Smith, McCrindle, Burnett, \& Campbell, 2001). Este último punto apoyaría la hipótesis del cambio conceptual producto del avance alcanzado a lo largo de la formación universitaria, sin embargo, aún no queda claro cuáles serían los pasos y las situaciones que propiciarían dichos cambios y cuáles serían los factores asociados a dicha restructuración formal (Lonka, Joram, \& Bryson, 1996).

\section{Las estrategias metacognitivas}

Desde el campo de la psicología cognitiva, se ha identificado a la metacognición como el proceso que permite organizar la información, facilitando la atención, la motivación, el aprendizaje, el recuerdo, la comprensión, tornándose, por tanto, como el proceso de base a las tareas intelectuales generales (Danserau, 1978; Decharms, 1972; Dweck, 1975; Mischel \& Baker, 1975; Nisbet \& Shucksmith, 1987; Valle et al., 1999; Vermunt, 1996, 1998).

En la década de los setenta del siglo pasado, Flavell (1979) acuña el termino, definiéndolo como el proceso de conocer el propio conocimiento. Una década después, se ha replanteado la definición del concepto, operacionalizándolo y dividiéndolo en dos áreas:

A) El conocimiento sobre los procesos cognitivos (saber qué), relativo a personas, estrategias o tareas.

B) La regulación de los procesos cognitivos (saber cómo), relacionada con la planificación, el control y la evaluación de los procesos cognitivos (Brown, Bransford, Ferrara, \& Campione, 1983).

Es sobre el área del "saber cómo", de tipo procedimental y estratégico, que la presente investigación se enfocará. Respecto de las estrategias metacognitivas, Schraw y Moshman (1995) han identificado tres tipos: la planificación (a), el control (b) y la evaluación (c). La planificación (a) incluye la selección de estrategias antes de la ejecución de la tarea y la selección de recursos adecuados para esta, por ejemplo: secuenciación de actividades y asignación de tiempo requerido. La estrategia de control (b) remite a la revisión que se realiza durante la tarea y a la autoevaluación durante la ejecución, por ejemplo: consideración del cómo se está ejecutando la tarea o variación de las estrategias que no están dando resultado. Y por último, la estrategia de evaluación (c) comprende la consideración de los productos o resultados finales de la tarea. Esta última estrategia establece una valoración de lo realizado y lo que ha quedado por mejorar de la actividad total (Schraw \& Moshman, 1995).

En cuanto a la investigación sobre metacognición en contextos universitarios, Boekaerts (1999) y Boekaerts y Niemivirta (2000) concluyen que la activación de la 
autorregulación del aprendizaje implicaría el uso de estrategias metacognitivas, requiriendo la motivación y el esfuerzo de los estudiantes como elemento mediacional. Esta necesidad adicional de motivación y esfuerzo por parte del estudiantado ocurriría por las características propias de los contextos educativos convencionales, es decir, aquellos que tienden a centrar el aprendizaje en el docente. En cuanto a la relación entre nivel de pericia y estrategias metacognitivas, Nickerson, Perkins y Smith (1985) plantean que los expertos no solo presentan mejor manejo de conocimiento que los novatos, sino que reconocen que saben más, empleando de mejor manera el conocimiento, en términos de organización, accesibilidad y adquisición de nuevos aprendizajes (Echeverría, Mateos, Pozo, \& Scheuer, 2001). Respecto de esto, algunas investigaciones en el contexto universitario, han señalado que la falta de conocimiento en el dominio específico de la psicología sería una de las principales razones, por la cual estudiantes noveles serían metacognitivamente menos hábiles que los expertos, produciendo así un conocimiento fragmentado y menos comprensivo (Martínez, 1999, 2004).

A partir de estos antecedentes, la presente investigación tiene como objetivo determinar la influencia del nivel de pericia en el dominio específico de la psicología y de las estrategias metacognitivas sobre los tipos de concepción de aprendizaje en estudiantes universitarios de psicología chilenos, considerando como base el estudio de Martínez (2007) con estudiantes universitarios españoles. A partir de esto, la originalidad de este trabajo reside en identificar el tipo y grado de influencia de ambas variables sobre el desarrollo de las concepciones de aprendizaje en estudiantes universitarios chilenos. Por tanto, se busca contrastar las siguientes hipótesis: (1) el nivel de pericia en psicología se asocia e influye sobre el tipo de concepción de aprendizaje, (2) las estrategias metacognitivas se asocian e influyen sobre el tipo de concepción de aprendizaje y (3) ambas variables se asocian e influyen sobre el tipo de concepción de aprendizaje.
Bajo esta propuesta de análisis, Brew (2001) ha planteado la necesidad de realizar mayor investigación en torno al modelo del cambio conceptual y el posible establecimiento de relaciones explicativas entre esta y el uso de estrategias de enseñanza, ampliando así el dominio explicativo sobre el aprendizaje universitario y sus condiciones.

\section{Metodología}

\section{Participantes}

Se utilizó un muestreo no probabilístico, al seleccionar, en primer lugar, las dos únicas universidades que imparten la Carrera de Psicología en la ciudad de Arica; y en segundo lugar, al seleccionar solo aquellos estudiantes que cumplieran con los criterios de inclusión muestral, establecidos de acuerdo con el nivel de pericia. Participaron en el estudio 184 estudiantes universitarios pertenecientes a la Carrera de Psicología. Estos se distribuyeron según el nivel de pericia que presentaban (Baja $=84$; Media $=59$ y Alta $=41)$. Los participantes cursaban el primer semestre (pericia baja), quinto semestre (pericia media) y noveno semestre (pericia alta) del primer semestre del año académico de las facultades de Psicología de la Universidad de Tarapacá y Universidad Santo Tomás. La edad promedio de los(as) estudiantes era de 22.42 años con una desviación estándar de 4.82. En cuanto al sexo de los participantes, 59 eran hombres (32.1\%), mientras 125 eran mujeres (67.9\%).

\section{Instrumentos y variables}

Los instrumentos y variables operacionalizadas fueron los siguientes:

Inventario de concepciones de aprendizaje (CONAPRE): inventario de autorreporte que evalúa concepción de aprendizaje. Instrumento diseñado y validado el año 1999 por Martínez Fernández. El cuestionario consta de 15 ítems, aunque inspirado inicialmente en la categorización de Säljö (1979), fue reformulado 
más tarde, tras un análisis factorial, en tres factores que corresponden a cada una de las categorías definidas por Pozo y Scheuer (1999) para la concepción de aprendizaje: directiva, interpretativa y constructivista. El formato de respuesta de la encuesta debe ser valorado en una escala que va desde uno (Nunca) a cinco (Siempre). Con los puntajes obtenidos se realizó el análisis de fiabilidad de escala, tanto por factor como por escala completa. Respecto de las confiabilidades por factor, se obtuvo un alfa de Cronbach de 0.73 para el factor directivo, 0.59 para el factor interpretativo y de 0.77 para el factor constructivista, mientras que a nivel general la confiabilidad obtenida fue de 0.71 .

Inventario sobre estrategias metacognitivas de aprendizaje: se utilizó el cuestionario de autoreporte de O'Neil y Abedi (1996). El instrumento se divide en dos factores: 1) planificación y 2) evaluación-control. Este instrumento posee veinte reactivos que se contestan de acuerdo con una escala que va desde uno (Nunca) a cinco (Siempre). Con los puntajes obtenidos, se realizó el análisis de fiabilidad de escala, tanto por factor como por el instrumento completo. Respecto de las confiabilidades por factor, se obtuvo un alfa de Cronbach de 0.76 para el factor planificación y de 0.82 para el factor control evaluación, mientras que a nivel general la confiabilidad obtenida fue de 0.80 (alpha de Cronbach).

Nivel de pericia: esta variable se operacionalizó en función del semestre que cursaba el estudiante. La Carrera de Psicología, en ambas universidades, contempla un total mínimo de 11 semestres. En el momento de la realización del estudio, correspondiente al primer semestre del año académico, se seleccionaron 84 estudiantes que se encontraban cursando el primer semestre (45.7\%), los cuales fueron codificados como nivel de pericia bajo; 59 estudiantes se encontraban cursando el quinto semestre (32.1 $\%)$, los cuales fueron codificados como nivel de pericia medio, y por último 41 estudiantes se encontraban cursando el noveno semestre (22.3 $\%)$, los cuales fueron codificados como nivel de pericia alto.

\section{Procedimiento}

En primer lugar, se identificaron y seleccionaron las dos universidades que impartían la Carrera de Psicología en la ciudad de Arica. Tras la obtención de los permisos correspondientes, con ambas direcciones de escuela, se estableció una reunión informativa con los profesores de las asignaturas con mayor cantidad de estudiantes inscritos de primer, quinto y noveno semestre, respectivamente. En cuanto a la producción de datos, se entregó a cada uno de los estudiantes cuadernillos con los instrumentos descritos, consignando el nivel cursado y los antecedentes sociodemográficos generales. El tiempo de respuesta de los cuadernillos fue de 15 minutos. Tras la aplicación del instrumento, se procedió a la digitación de los datos y su posterior análisis, utilizando el programa IBM SPSS Statistics 18. Se efectuaron análisis de correlación de Pearson, análisis de varianza (ANOVA) con prueba de comparaciones múltiples y análisis de regresión múltiple con el método de extracción de pasos sucesivos. Respecto de la dimensión ética de la investigación, con la finalidad de resguardar la confidencialidad de la información obtenida, el cuadernillo de cuestionarios fue respondido de forma voluntaria, anónima y supervisada por el investigador responsable. Por último, la investigación no contó con financiamiento externo.

\section{Resultados}

Asociaciones entre las variables centrales del estudio

Como primer objetivo se indagó sobre el grado de asociación, por medio del coeficiente de correlación lineal de Pearson, entre los puntajes obtenidos en estrategias metacognitivas y los tres tipos de concepción de aprendizaje. De acuerdo con lo observado, las estrategias metacognitivas presentan una relación negativa y estadísticamente significativa con la dimensión directiva $r(184)=-.015, p=0.035$; por el contrario, mostró una relación positiva y

| Universitas Psychologica | V. i6 | No. 2 | Abril-Junio | 20 I7 | 
significativa con la dimensión interpretativa $r(184)=0.61, p=0.000$ y la dimensión constructiva $r(184)=0.65, p=0.000$.

\section{Análisis de varianza (ANOVA)}

Como segundo objetivo, se analizó la existencia de diferencias significativas entre los tres tipos de concepción de aprendizaje: directivo, interpretativo y constructivista, en función de los tres niveles de pericia: bajo, medio y alto. Para esto, se utilizó el análisis de varianza ANOVA de un factor sobre cada tipo de concepción de aprendizaje.

Concepción directiva: para la concepción directiva, los 84 participantes de pericia baja presentaron una puntuación media de 15.29 $(D E=2.11)$, los 59 participantes de pericia media presentaron una media de 11.86 (DE $=3.06)$ y los 41 participantes de pericia alta tuvieron un promedio de $10.73(\mathrm{DE}=$ 3.31). El estadístico de Levene $(6.73, p=$ 0.002) permite concluir que las varianzas de los tres grupos no son iguales. Se obtuvo una diferencia estadísticamente significativa de concepción directa sobre los niveles de pericia, $F$ $(2.181)=48.07, p=0.000, \eta_{p}^{2}=0.347,95 \%$ ICs $[14.83,15.74]$, [11.07, 12.66] y $[9.68,11.78]$. La prueba de Games-Howell reveló que las diferencias se producen entre el grupo de pericia baja y los otros dos $(p=0.000)$, mientras que el grupo de pericia media no alcanzó a diferenciarse significativamente del grupo de pericia alta $(p=$ 0.199).

Concepción interpretativa: para la concepción interpretativa, los 84 participantes de pericia baja presentaron una puntación media de 20.01 $(D E=4.08)$, los 59 participantes de pericia media presentaron una media de 22.59 (DE $=3.02)$ y los 41 participantes de pericia alta tuvieron un promedio de $22.20(\mathrm{DE}=3.49)$. El estadístico de Levene $(3.16, p=0.045)$ permite concluir que las varianzas de los tres grupos no son iguales. Se obtuvo una diferencia estadísticamente significativa de concepción directa sobre los niveles de pericia, $F(2.181)=$ $10.19, p=0.000, \eta_{p}^{2}=0.101,95 \%$ ICs $[19.13$,
20.90], [21.81, 23.38] y [21.09, 23.30]. La prueba de Games-Howell reveló que las diferencias se producen entre el grupo de pericia baja, frente a pericia media $(p=0.000)$ y alta $(p=0.007)$, mientras que el grupo de pericia media no alcanzó a diferenciarse significativamente del grupo de pericia alta $(p=0.825)$.

Concepción constructiva: para la concepción constructiva, los 84 participantes de pericia baja presentaron una puntación media de 15.67 (DE $=3.92)$; los 59 participantes de pericia media presentaron una media de $16.15(D E=3.66)$ y los 41 participantes de pericia alta tuvieron un promedio de $19.63(\mathrm{DE}=3.03)$. El estadístico de Levene $(1.19, p=0.304)$ permite concluir que las varianzas de los tres grupos son iguales. Se obtuvo una diferencia estadísticamente significativa de concepción directa sobre los niveles de pericia, $F(2,181)=17.14, p=0, \eta_{p}^{2}=0.159,95$ $\%$ ICs [14.81, 16.52], [15.20, 17.11] y [18.67, 20.59]. La prueba de Bonferroni reveló que las diferencias se producen entre el grupo de pericia alta frente a pericia baja $(p=0)$ y media $(p=0)$, mientras que el grupo de pericia baja no alcanzó a diferenciarse significativamente del grupo de pericia media $(p=0.730)$.

\section{Análisis de regresión con el método de extracción de pasos sucesivos}

Para determinar el grado de influencia de las dos principales variables independientes del estudio, es decir, 1) Estrategias metacognitivas y 2) Nivel de pericia sobre los tres tipos de concepciones de aprendizaje, se utilizó el análisis de regresión múltiple con el método de extracción de pasos sucesivos (stepwise), con el fin de que solo ingresen, en orden de importancia, las variables significativas estadísticamente.

Directiva: los puntajes en la concepción directiva fueron regresados considerando las estrategias metacognitivas y el nivel de pericia, del cual solo esta última fue considerada en el modelo final. El nivel de pericia explicó $31 \%$ de la varianza de los puntajes de concepción de aprendizaje $(R 2=0.32)$, siendo muy significativo, $F(1,182)=86.70 p=0$. 
Por último, el nivel de pericia, $\beta=-0.56, p$ $=0,95 \%$ IC $[-2.92,-1.90]$, demostró efectos significativoss sobre la concepción de aprendizaje directiva.

Interpretativa: en segundo lugar, los puntajes en la concepción interpretativa fueron regresados considerando las estrategias metacognitivas y el nivel de pericia, del cual solo las estrategias metacognitivas fueron ingresadas en el modelo final. Las estrategias metacognitivas explicaron $37 \%$ de la varianza de los puntajes de concepción de aprendizaje $(R 2=0.37)$, siendo muy significativo, $F(1,182)=109.54 \mathrm{p}=0$. Por último, el nivel de pericia, $\beta=0.61, p=0$, $95 \%$ IC $[0.169,0.247]$, demostró efectos significativos sobre la concepción de aprendizaje interpretativa.

Constructivista: por último, los puntajes en la concepción constructivista fueron regresados considerando las estrategias metacognitivas y el nivel de pericia, del cual solo las estrategias metacognitivas fueron ingresadas en el modelo final. Las estrategias metacognitivas explicaron $43 \%$ de la varianza de los puntajes de concepción de aprendizaje $(R 2=0.43)$, siendo muy significativo, $F(1,182)=139.82 p=0$. Por último, el nivel de pericia, $\beta=0.65, p=0$, $95 \%$ IC $[0.194,0.271]$, demostró efectos significativos sobre la concepción de aprendizaje constructivista.

\section{Discusión}

En la presente investigación, se analizó el grado de asociación entre el tipo de concepción de aprendizaje, el uso de estrategias metacognitivas y el nivel de pericia en psicología de estudiantes universitarios chilenos. Los principales resultados evidencian que tanto las estrategias metacognitivas de aprendizaje como el nivel de pericia en psicología presentaron una relación significativa entre los tres tipos de concepción de aprendizaje. Sin embargo, en un análisis detallado sobre cada tipo de concepción, aquellas que presentan un mayor grado de elaboración, interpretativo y constructivo, tendieron a estar mayormente influenciadas por el uso de estrategias metacognitivas de aprendizaje. Los resultados similares han sido reportados por Säljö (1979) y Vermuntt (1998), al identificar la presencia de relaciones significativas entre el uso de estrategias de autorregulación por parte del estudiantado y la respectiva posesión de una concepción constructivista del aprendizaje por parte de estos.

En cuanto al nivel de pericia en psicología, de acuerdo con la evidencia obtenida, los estudiantes que presentaron altas puntuaciones en los tipos de aprendizaje interpretativo y constructivo tendieron a estar cursando el tercer y quinto año de la Carrera de Psicología. Estos resultados se condicen con la evidencia entregada por Alexander et al. (1998) y BoultonLewis et al. (2001).

Sin embargo, al analizar el grado de influencia de ambas variables independientes sobre los tipos aprendizaje de mayor complejidad y elaboración, fueron las estrategias metacognitivas las que tuvieron mejor peso predictivo sobre ambas. Esto quiere decir que, si bien inicialmente existe una relación asociativa entre concepciones de aprendizaje, como categoría general, y niveles de pericia en psicología más avanzados, tercer y quinto año respectivamente, serían las estrategias metacognitivas de planificación, evaluación y control las que predecirían mejor el avance del aprendizaje directo hacia uno de carácter más interpretativo o constructivo. Este resultado se condice con investigaciones que señalan que los estudiantes expertos, en algún dominio específico, no solo sabrían más sino que también conocerían los mecanismos vinculados a una mejor adquisición del conocimiento. En síntesis, este grado de experticia parecería influir, a su vez, en el aumento de la capacidad para resolver problemas en el dominio particular de la psicología, favoreciendo así los procesos de cambio conceptual (Nickerson et al., 1985).

Por otro lado, los estudiantes noveles que cursan el primer año de psicología presentaron una concepción de aprendizaje más directiva y una escasa utilización de estrategias metacognitivas. Por esto, para la concepción directiva, el bajo dominio específico en psicología, al encontrarse en la etapa 
inicial de la carrera universitaria, se mostró como el principal predictor, evidenciando el predominio de estrategias de corte memorístico o directo por parte del estudiantado de primer año, reproduciendo así la información de los docentes y adoptando un rol pasivo-receptivo en el aula. Respecto de este último punto, las posteriores investigaciones debiesen profundizar en la relación, aplicada al contexto universitario, entre las estrategias de enseñanza y los criterios de evaluación docente, dado que ambos irían de la mano con el tipo de aprendizaje promovido (Villalobos et al., 2009; Marshall, Summer, \& Woolnough, 1999).

En relación con este último punto, como aspecto por considerar en posteriores investigaciones del área, sería relevante indagar sobre la posible relación entre el tipo de concepción directiva y rendimiento académico, debido a las altas tasas de deserción al término del primer año universitario, equivalente a 19 $\%$ aproximadamente en universidades estatales y $22 \%$ aproximadamente en las universidades privadas, de acuerdo con datos del Consejo Superior de Educación (Centro de Microdatos, 2008).

Presentado el antecedente sobre la deserción universitaria en el primer año, iqué pasaría si a los estudiantes noveles de Psicología, es decir, de primer año, se les enseñara diversas estrategias metacognitivas antes de la entrega de los contenidos establecidos para su formación profesional? ¿Podría favorecer la enseñanza de estrategias metacognitivas, el avance hacia concepciones de aprendizaje de mayor complejidad? (Hernández, 2002). Estas son solo algunas de los interrogantes que debieran explorar las futuras investigaciones, aplicables no solo al campo de la psicología, sino a todo campo investigativo que se preocupe y ocupe del estudio del desarrollo conceptual y del aprendizaje en general.

\section{Agradecimientos}

El trabajo se realizó en el marco del programa de Magister en Ciencias Sociales Aplicadas perteneciente a la Universidad de Tarapacá.

\section{Referencias}

Alexander, P., Murphy, K., Guan, J., \& Murphy, P. (1998). How students and teachers in Singapore and the United States conceptualize knowledge and beliefs: Positioning learning within epistemological frameworks. Learning and Instruction, 8(2), 97-116. https://doi.org/10.1016/S0959-475 2(97)00004-2 .

Boekaerts, M. (1999). Self-regulated learning: Where we are today. International Journal of Educational Research, 31(6), 445-457. https://doi.org/10.1016/S0883-03 55(99)00014-2 .

Boekaerts, M., \& Niemivirta, M. (2000). Selfregulated learning: Finding a balance between learning goals and ego-protective goals. En M. Boekaerts, P. Pintrich, \& M. Zeidner (Eds.), Handbook of selfregulation: Theory, research, and applications (pp. 417-450). San Diego, CA: Academic Press.

Boulton-Lewis, G., Marton, F., Lewis, D., \& Wilss, L. (2000). Aboriginal and Torres Strait Islander university students' conceptions of formal learning and experiences of informal learning. Higher Education, 39(4), 469-488. https://doi.org/1 0.1023/A:1004060422023.

Boulton-Lewis, G., Smith, D., McCrindle, A., Burnett, P., \& Campbell, K. (2001). Secondary teachers' conceptions of teaching and learning. Learning and instruction, 11 (1), 35-51. https://doi.org/10. 1016/S0959-4752(00)00014-1 .

Brew, A. (2001). Conceptions of research: A phenomenographic study. Studies in Higher Education, 26(3), 271-285. https://doi.org/1 0.1080/03075070120076255 
Brown, A., Bransford, J., Ferrara, R., \& Campione, J. (1983). Learning, remembering, and understanding. En J. Flavell, \& E. Markman (Eds.), Handbook of child psychology: Child development (pp.77-166). New York: Wiley.

Carey, S. (1985a). Conceptual change in childhood. Cambridge, MA: Bradford Books, MIT Press.

Carey, S. (1985b). Are children fundamentally different thinkers and learners from adults? En S. Chipman, J. Segal, \& R. Glaser (Eds.), Thinking and learning skills (pp. 485-517). Hillsdale, NJ: Erlbaum. Reprinted by Open University Press: Open University Readings in Cognitive Development.

Centro de Microdatos (2008). Informe final: estudios sobre causas de la deserción universitaria. Santiago de Chile: Universidad de Chile. Recuperado de http://www.opech.cl/educsuperior/politi caacceso/informefinalcausasdesercionuniv ersitaria.pdfLinks] opech.cl/educsuperior/p olitica_acceso/informe_final_causas_deser cion_universitaria.pdf

Danserau, D. (1978). The development of a learning strategy curriculum. En H. O'Neil (Ed.), Learning strategies (pp. 1-29). New York: Academic Press.

Decharms, R. (1972). Personal causation training in the schools. Journal of Applied Social Psychology, 2(2), 95-113. https://doi.org/10. 1111/j.1559-1816.1972.tb01266.x

Dweck, C. (1975). The role of expectations and attributions in the alleviation of learned helplessness. Journal of Personality and Social Psychology, 31(4), 674-684. https://doi.org/ 10.1037/h0077149

Dykstra, D., Boyle, F., \& Monarch, I. (1992). Studying conceptual change in learning physics. Science Education, 76(6), 615-652. https://doi.org/10.1002/sce.3730760605

Echeverría, M., Mateos, M., Pozo, J., \& Scheuer, N. (2001). En busca del constructivismo perdido: concepciones implícitas sobre el aprendizaje. Estudios de Psicología, 22(2), 155-173. https://doi.org/10.1174/02109390 1609479
Flavell, J. (1979). Metacognition and cognitive monitoring: A new area of cognitive developmental inquiry. American Psychologist, 34(10), 906-911. https://doi.or g/10.1037/0003066X.34.10.906

Glaser, R. (1982). Instructional psychology: Past, present, and future. American Psychologist, 37(3), 292-305. https://doi.org/10.1037/00 03-066X.37.3.292

Hernández Pina, F. (2002). Docencia e investigación en educación superior. Revista de Investigación Educativa, 20(2), 271-301.

Hewson, P., \& Thorley, N. (1989). The conditions of conceptual change in the classroom. International Journal of Science Education, 11 (5), 541-553. https://doi.org/1 0.1080/0950069890110506

Kuhn, D., \& Lao, J. (1998). Contemplation and conceptual change: Integrating perspectives from social and cognitive psychology. Developmental Review, 18(2), 125-154. https://doi.org/10.1006/drev.1997 .0449

Limón, M. (2001). On the cognitive conflict as an instructional strategy for conceptual change: A critical appraisal. Learning and Instruction, 11(4), 357-380. https://doi.org/ 10.1016/S0959-4752(00)00037-2

Lonka, K., Joram, E., \& Bryson, M. (1996). Conceptions of learning and knowledge: Does training make a difference? Contemporary Educational Psychology, 21 (3), 240-260. https://doi.org/10.1006/ceps.1996 .0021

Máiquez, M., Rodrígo, M., Capote, C., \& Vermaes, I. (2000). Aprender en la vida cotidiana: un programa experiencial para padres. Madrid: Visor.

Marshall, D., Summer, M., \& Woolnough, B. (1999). Students' conceptions of learning in an engineering context. Higher Education, 38(3), 291-309. https://doi.org/1 0.1023/A:1003866607873

Martínez Fernández, R. (1999). Aprendizaje en la Universidad: desafíos para el siglo XXI. Del énfasis en los productos al énfasis en los procesos. Revista Latinoamericana de Psicología, 31 (3), 491-504. 
Martínez Fernández, R. (2004). Concepción de aprendizaje, metacognición y cambio conceptual en estudiantes universitarios de psicología (Tesis doctoral, Universitat de Barcelona, Barcelona, España). Recuperado de http://diposit.ub.edu/dspac e/handle/2445/42698/

Martínez Fernández, R. (2007). Concepción de aprendizaje y estrategias metacognitivas en estudiantes universitarios de psicología. Anales de Psicología, 23(1), 7-16.

Mischel, W., \& Baker, N. (1975). Cognitive appraisals and transformations in delay behavior. Journal of Personality and Social Psychology, 31(2), 254 - 261. https://doi.org $/ 10.1037 / \mathrm{h} 0076272$

Nickerson, R., Perkins, D., \& Smith, E. (1985). The teaching of thinking. Hillsdale, NJ: Lawrence Erlbaum Associates.

Nisbet, J., \& Shucksmith, J. (1987). Estrategias de aprendizaje. Madrid: Santillana.

O’Neil, H., \& Abedi, J. (1996). Reliability and validity of a state metacognitive inventory: Potential for alternative assessment. The Journal of Educational Research, 89(4), 234-245. https://doi.org/10.1080/00220671 .1996 .9941208

Pintrich, R., Marx, R., \& Boyle, R. (1993). Beyond cold conceptual change: The role of motivational beliefs and classroom contextual factors in the process of conceptual change. Review of Educational research, 63(2), 167-199. https://doi.org/10. 3102/00346543063002167

Pozo, J. (2003). Adquisición de conocimiento: cuando la carne se hace verbo. Madrid: Morata.

Pozo, J. \& Scheuer, N. (1999). Las concepciones sobre el aprendizaje como teorías implícitas. En J. Pozo, \& C. Monereo (Eds.), El aprendizaje estratégico (pp. 87-108). Madrid: Santillana.

Pozo, J., \& De La Cruz, M. (2003). Concepciones sobre el currículo universitario: icentrado en los contenidos o centrado en los alumnos? En C. Monereo, \& J. Pozo (Eds.), La universidad ante la nueva cultura educativa: enseñar y aprender para la autonomía (pp. 141-154). Madrid: Síntesis.

Reif, F., \& Larkin, J. (1991). Cognition in scientific and everyday domains: Comparison and learning implications. Journal of Research in Science Teaching, 28(9), 733-760. https://doi.org/10.1002/tea .3660280904

Rodríguez Moneo, M., \& Carretero, M. (1996). Adquisición de conocimiento y cambio conceptual: implicaciones para la enseñanza de las ciencias. En M. Carretero (Ed.), Construir y enseñar: las ciencias experimentales (pp. 47-73). Buenos Aires: Aique.

Säljö, R. (1979). Learning in the learners' perspective. I. Some common sense conceptions. Reports from the Institute of Education, University of Göteborg, 76, 656-658.

Schommer, M. (1993). Epistemological development and academic performance among secondary students. Journal of Educational Psychology, 85(3), 406-411. htt ps://doi.org/10.1037/0022-0663.85.3.406

Schommer, M., Calvert, C., Gariglietti, G., \& Bajaj, A. (1997). The development of epistemological beliefs among secondary students: A longitudinal study. Journal of Educational Psychology, 89(1), 37-40. https: //doi.org/10.1037/0022-0663.89.1.37

Schraw, G., \& Moshman, D. (1995). Metacognitive theories. Educational Psychology Review, 7(4), 351-371. https://do i.org/10.1007/BF02212307

Tynjälä, P. (1997). Developing education students' conceptions of the learning process in different learning environments. Learning and Instruction, 7(3), 277-292. https://doi.org/10.1016/S0959-47 $52(96) 00029-1$

Tynjälä, P. (1999). Towards expert knowledge? A comparison between a constructivist and a traditional learning environment in the university. International Journal of Educational Research, 31 (5), 357-442. https: //doi.org/10.1016/S0883-0355(99)00012-9 
Valle, A., González, R., Núñez, J., Vieiro, P., Gómez, M., \& Rodríguez, S. (1999). Un modelo cognitivo-motivacional explicativo del rendimiento académico en la universidad. Estudios de Psicología, 20(62), 77-100. https://doi.org/10.1174/02 109390260288631

Vermunt, J. (1996). Metacognitive, cognitive and affective aspects of learning styles and strategies: A phenomenographic analysis. Higher Education, 31 (1), 25-50. https://doi. org/10.1007/BF00129106

Vermunt, J. (1998). The regulation of constructive learning processes. British Journal of Educational Psychology, 68(2), 149-171. https://doi.org/10.1111/j.2044-82 79.1998.tb01281.x

Villalobos, E., Guerrero, M., Pérez, R., Avendaño, A., Ceballos, A., Ortiz, J., \& Parra, C. (2009). Estilos de aprendizaje y metodologías de enseñanza en estudiantes de obstetricia. Educación Médica, 12(1), 43-46.

White, R., \& Gunstone, R. (1989). Metalearning and conceptual change. International Journal of Science Education, 11(5), 577-586. https://doi.org/10.1080/09500698 90110509

\section{Notas}

* Artículo de investigación 